\title{
Numerical Studies of the Aerodynamic Features of Dead-end Entries with Side Junction
}

\author{
Yuri Govorukhin ${ }^{1,2}$, Victor Krivolapov ${ }^{1}$, Dmitry Paleev1 ${ }^{1,3}$, and Vyacheslav Portola ${ }^{4}$ \\ ${ }^{1}$ Federal State Institution "National Rescue Center" Ministry of Emergency Situations of Russia, \\ 654044, Novokuznetsk, Prospect Aviatorov, 54, Russia \\ ${ }^{2}$ Siberian State Industrial University, 654007, Novokuznetsk, Kirova Str., 42, Russia \\ ${ }^{3}$ Mining Institute of the Ural Branch of the Russian Academy of Sciences, 614007, Perm, Siberian \\ Str., 78a,Russian Federation \\ ${ }^{4}$ T.F. Gorbachev Kuzbass State Technical University, Department of an aerology, labor protection \\ and nature, 650000 Kemerovo, 28 Vesennya St., Russian Federation
}

\begin{abstract}
Investigations of aerodynamic processes occurring in dead-end short entriesaired by turbulent diffusion have been performed. The numerical simulation of the processes of air movement through the entry, flow stalling at the junction with the dead-end entry (for side junction), and the formation of vortices at the dead end have been carried out. The study has been done for a wide range of air flows submitted for computation of air consumption and for various geometric parameters of the dead-end entry. The sizes of the vortex structures and the flow rates in the dead endshave been determined. Based on the results of processing the simulation data, we obtained graphs of the dependences between the length of the ventilated zone of the dead end and its height and width.
\end{abstract}

\section{Introduction}

According to federal norms and rules in the field of industrial safety "Safety Rules for Coal Mines" ventilation of dead-end entries of gas mines with a length of not more than $6 \mathrm{~m}$ and non-gas mines up to $10 \mathrm{~m}$ [1] could be possible due to turbulent diffusion processes.Experimental studies of the processes of airflowpassage past dead-end entries were carried out in 40-50s (V.N. Voronin [2-4] and A.M. Karpov [2]). According to the results of these studies, the physics of aerodynamic processes was described, and V.N. Voronin introduced the concept of turbulent diffusion as a process of interaction of a turbulent free stream of fresh air and the mixture of air and mine gases surrounding this stream in a space that is subject to ventilation. A.M. Karpovobtained various stable turbulent zones depending on the location of the dead-end in relation to the entry: direct $(3 \div 5 b)$, side $(3 \div 4 b)$, reverse $(2 \div 3 b)$, where $b$ is the width of the dead-end entry.

The studies were conducted for the entries with small sections fixed by wooden supports, and their results for the mining conditions of modern mines can vary significantly.

To study the aerodynamic processes in dead-end entries, numerical modeling is performed. For this purpose, the open source OpenFOAM package (The OpenFOAM Foundation Ltd) [5-7] was used. To prepare the initial data, such as specifying the geometry of the computational domain, constructing a grid, and setting boundary conditions, a multi-block 
grid generator, blockMesh, was selected. This utility divides the computational domain into hexahedrons. The paradigm of creating mesh structures in OpenFOAM allows the use of degenerate forms of hexagons. For the numerical solution, the standard simpleFoam solver was chosen (the solver for stationary problems of an incompressible, turbulent flow; the SIMPLE algorithm is used - the Semi-Implicit Method for Pressure-Linked Equations of the Patankar method). Visualization and processing of the calculation results were performed using the ParaView postprocessor (supplied with free distributed OpenFOAM). ParaView provides visualization of scalar and vector fields, streamlines, isosurfaces, obtaining information about the desired field at a point or along a certain line, etc.

A Beowulf-type computing cluster has been created based on:

- 3 monoblockiRUs with Intel Core i5-3330 processors, with a total number of computing cores - 12 ;

- D-Link DGS-1005 / D switch with a packet data rate of up to 1 Gbit / s (Ethernet network).

The computational cluster made it possible to reduce the computation time compared to a single computer by decomposing the computational domain into parallel executable branches that exchange data over a connecting network. To evaluate the increase in productivity using a 'Beowulf' type computing cluster, air movement over a straight section of the mine working was calculated ( 25 thousand hexahedrons, the total number of iterations was 1000). The calculation results are summarized in Table 1.

Thus, the use of parallel algorithms for calculations using the OpenFOAM package on a computing cluster can reduce the calculation timesignificantly. Especially when creating computational areas of large sizes and using a fine mesh.

Table 1. Comparison of the calculations duration.

\begin{tabular}{|l|c|c|c|}
\hline Cores number, units & $\mathbf{1}$ & $\mathbf{2}$ & $\mathbf{4}$ \\
\hline Calculation time, sec. & 105.6 & 56.75 & 46.02 \\
\hline $\begin{array}{l}\text { Productivity increase, } \\
\text { times }\end{array}$ & - & 1.86 & 2.29 \\
\hline
\end{tabular}

\section{Mathematical setting of the problem}

For a mathematical description of a turbulent incompressible, isothermal, single-phase flow in a stationary formulation, the Navier-Stokes differential equations averaged over the Reynolds number (RANS equations) are used.

The general scheme of boundary conditions for mathematical models of local resistance circuits is shown in Figure 1, the Dirichlet boundary conditions (1st kind) and von Neumann (2nd kind) are used. The problem is solved in two-dimensional space.

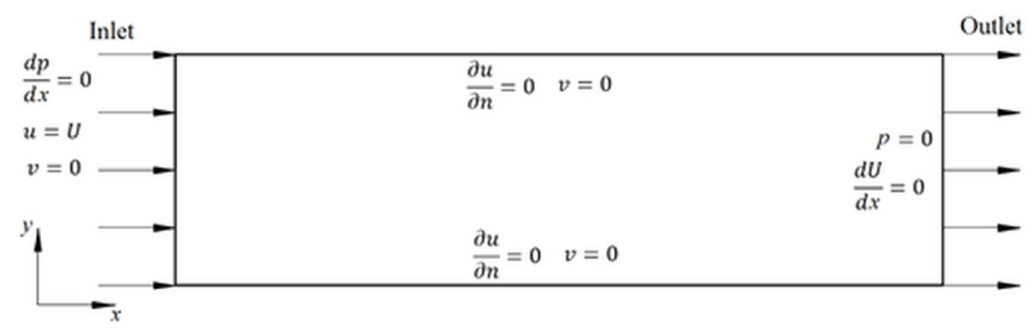

Fig. 1. The boundary conditions of the models under consideration for $p$ (gauge pressure) and for $\mathrm{U}$ (flow velocity vector). 


\section{Modeling}

To study the aerodynamic features of dead ends (side junction), a basic model is created, shown in Figure 2. The model consists of four block super elements, each of which the blockMesh grid generator de-composes into hexahedrons. The numbers in Figure 2 indicate the vertices of the super elements. The length and width of the entry (excluding the junction dimensions) are $80 \mathrm{~m}$ and $6 \mathrm{~m}$, respectively, for all series of experiments. The length of the dead-end entry is $20 \mathrm{~m}$.

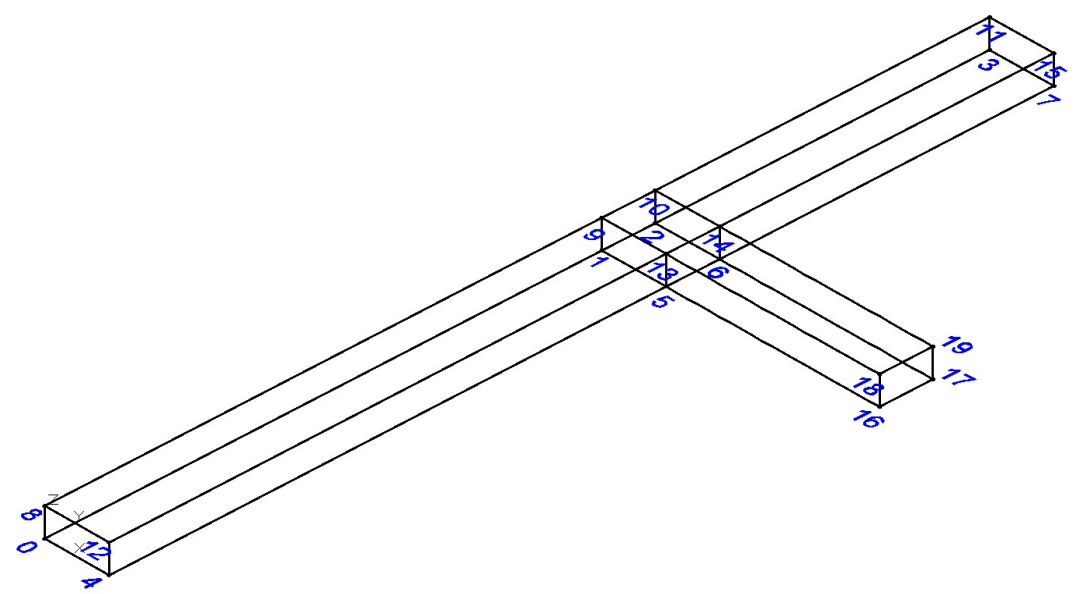

Fig. 2. The basic model created to study the aerodynamic features of dead ends.

Numerical simulation was performed for the conditions shown in table 2. In total, 90 series of experiments have been carried out.

Table 2. The Matrix of the initial conditions for which the simulation has been done.

\begin{tabular}{|c|c|c|c|c|c|c|c|c|c|c|c|}
\hline Dead-end & Dead-end & \multicolumn{10}{|c|}{ Air flow rates in the entry, $\mathrm{m} / \mathrm{s}$} \\
\hline \multirow{3}{*}{2.0} & 3.0 & \multirow{9}{*}{0.15} & \multirow{9}{*}{0.5} & \multirow{9}{*}{1.0} & \multirow{9}{*}{1.5} & \multirow{9}{*}{2.0} & & & & & \\
\hline & 4.8 & & & & & & & & & & \\
\hline & 6.0 & & & & & & & & & & \\
\hline \multirow{3}{*}{2.5} & 3.0 & & & & & & & & & & \\
\hline & 4.8 & & & & & & 2.5 & 3.0 & 4.0 & 5.0 & 6.0 \\
\hline & 6.0 & & & & & & & & & & \\
\hline \multirow{3}{*}{3.0} & 3.0 & & & & & & & & & & \\
\hline & 4.8 & & & & & & & & & & \\
\hline & 6.0 & & & & & & & & & & \\
\hline
\end{tabular}

Fig. 3 shows the distribution of flow rates in the dead-end entry for different air flow rates in the entry (data for $\mathrm{S}=12 \mathrm{~m}^{2}$, height $-2.5 \mathrm{~m}$, width $-4.8 \mathrm{~m}$ ). Designations are used: black vertical lines - vortex boundaries (Figure 4), red horizontal, dashed line - minimum flow rate $(0.15 \mathrm{~m} / \mathrm{s})$. 


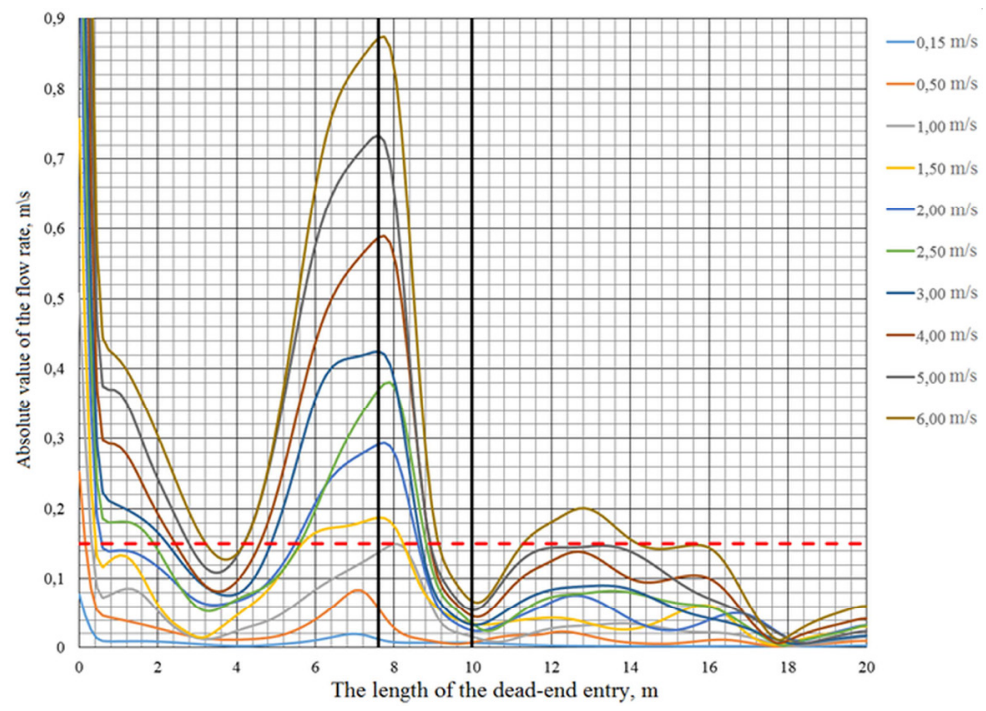

Fig. 3. Distribution of air flow rates in thedead-end entry at various flow rates in the entry (along the axis of the mine).

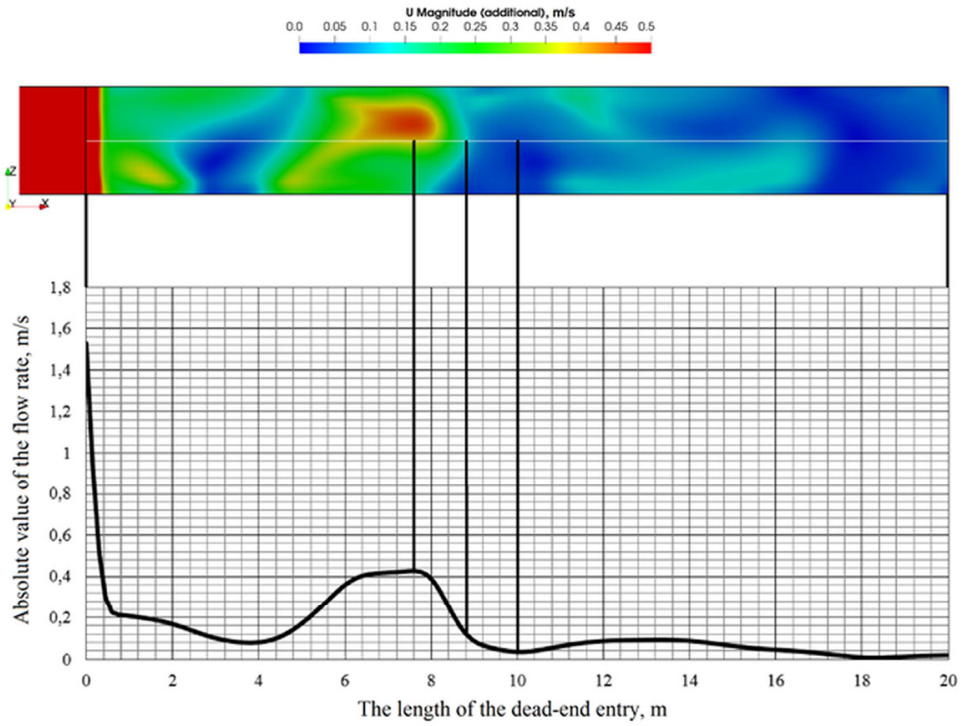

Fig. 4. Scheme explaining the principle of determining the size of the first vortex in a dead end entry (along the axis of the mine).

Figures 5-7 show streamlines in a dead-end entry (vortex dimensions) for the entry of $2.5 \mathrm{~m}$ high and of $3.0 \mathrm{~m}, 4.8 \mathrm{~m}, 6.0 \mathrm{~m}$ wide, the average flow rate along the entry is $3 \mathrm{~m} / \mathrm{s}$. Two color scales were used to indicate flow rates. "U Magnitude (main), $\mathrm{m} / \mathrm{s}$ " - for the entry. "U Magnitude (additional), $\mathrm{m} / \mathrm{s}$ " - for the dead-end entry. 


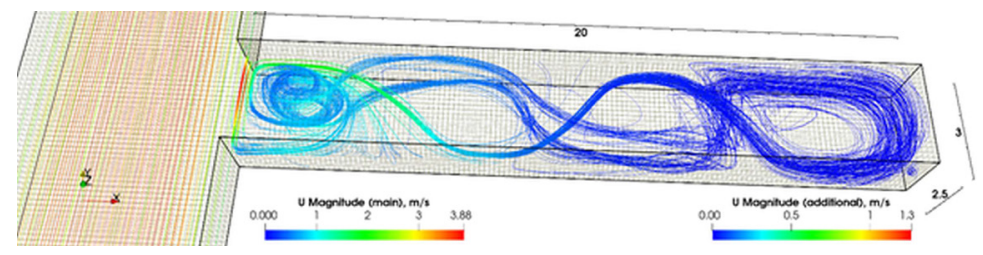

Fig. 5. Current lines for the entry width of $3 \mathrm{~m}\left(\mathrm{~S}=7.5 \mathrm{~m}^{2}\right)$.

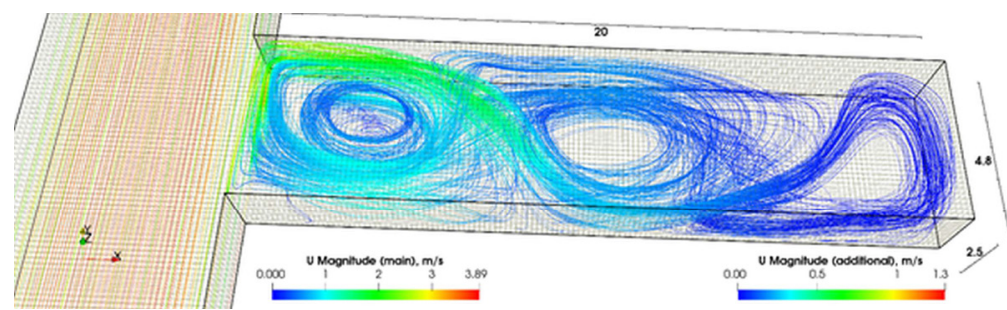

Fig. 6. Current lines for the entry width of $4.8 \mathrm{~m}\left(\mathrm{~S}=12.0 \mathrm{~m}^{2}\right)$.

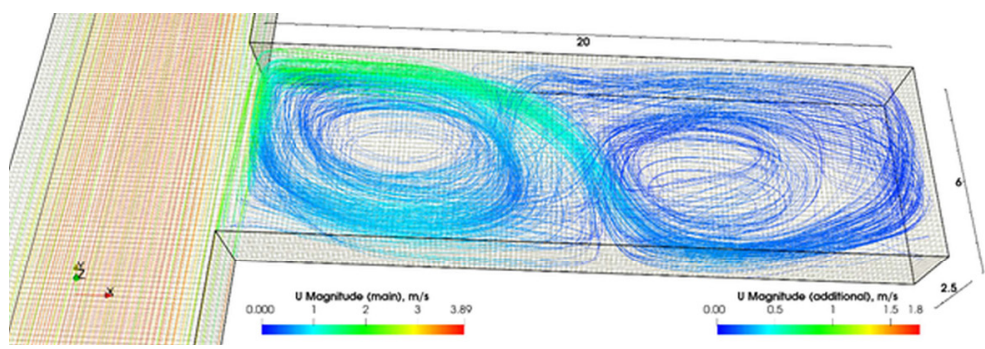

Fig. 7. Current lines for the entry width of $6.0 \mathrm{~m}\left(\mathrm{~S}=15.0 \mathrm{~m}^{2}\right)$.

\section{Results and Conclusions}

Figures 8 and 9 show the obtained dependences of the length of the ventilated zone onits width and height, respectively.

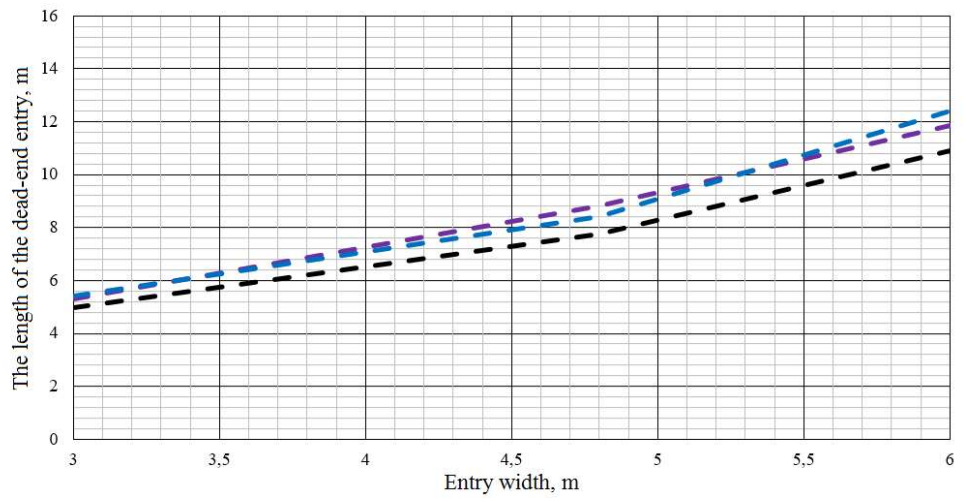

Fig. 8. Dependence of the average length of the vortex zone on the width of the dead-end entry with side junction: black lines $-2.0 \mathrm{~m}$ high; purple lines $-2.5 \mathrm{~m}$ high; blue lines $-3.0 \mathrm{~m}$ high. 


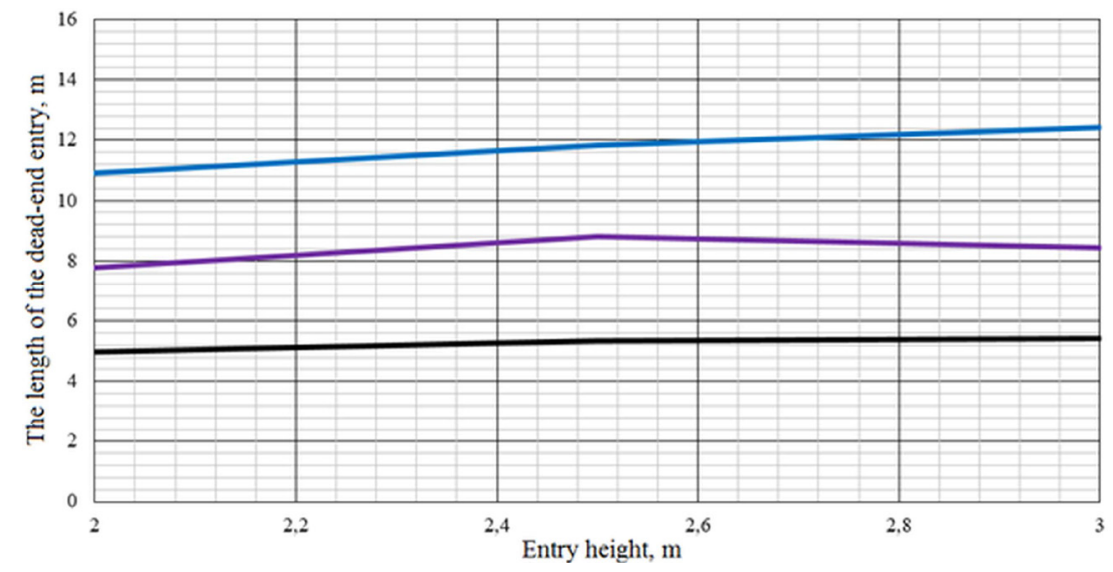

Fig. 9. Dependence of the average length of the vortex zone on the height of the dead-end entry with side junction: black lines - $3.0 \mathrm{~m}$ wide; purple lines - $4.8 \mathrm{~m}$ wide; blue lines - $6.0 \mathrm{~m}$ wide.

According to the results of numerical studies, it was found outthat for the dead-end entry with side junction:

- the requirements of federal norms and rules in the field of industrial safety [1] are generally justified, but only for those cases when the flow rate in the entry is over $1 \mathrm{~m} / \mathrm{s}$. This ensures a minimum flow rate in the dead end of $0.15 \mathrm{~m} / \mathrm{s}$ at distances from the mouth to $6 \mathrm{~m}$ (for gas mines);

- the size of the zones ventilated due to turbulent diffusion, mainly depends on the width of the entry, the entry height is not critical;

- vortex flows are complex, a flat free stream is formed (according to V.N. Voronin), which airs the dead end after the eight-shaped pattern.

\section{References}

1. Order of the Federal Service for Ecological, Technological and Nuclear Supervision dated November 19, 2013 No. 550 "On approval of the Federal Norms and Rules in the Field of Industrial Safety "Safety Rules in Coal Mines" (Ministry of Justice of Russia, Moscow, 2018).

2. A.A. Skochinsky, V.B. Komarov, Mine ventilation (Coal Press, Moscow, 1959)

3. V.N. Voronin, Fundamentals of mine aerodynamics (Coal Press, Moscow, 1959)

4. K.Z. Ushakov, A.S. Burchakov, L.A. Puchkov, I.I. Medvedev, Aerology of mining enterprises: a textbook for universities (Nedra, Moscow, 1987)

5. A.Yu. Popov, On the use of freely distributed software for solving problems of computational fluid dynamics (Publishing House of MSTU, Moscow, 2011)

6. Yu.M. Govorukhin, V.G. Krivolapov, D.Yu. Paleev, The use of CFD packages for solving the problems of mine ventilation and training of mining engineers (Publishing House of SibGIU, Novokuznetsk, 2019)

7. Yu.M. Govorukhin, V.N. Fryanov, D.Yu. Paleev, Bulletin of Tula State University. Earth sciences, 2, 288-299 (2019) 